Article

\title{
Flavonoids as Novel Efflux Pump Inhibitors and Antimicrobials Against Both Environmental and Pathogenic Intracellular Mycobacterial Species
}

\author{
Julia Solnier ${ }^{1,2}$, Liam Martin ${ }^{2}\left(\mathbb{D}\right.$, Sanjib Bhakta ${ }^{2}\left(\mathbb{D}\right.$ and Franz Bucar ${ }^{1, *(1)}$ \\ 1 Institute of Pharmaceutical Sciences, Department of Pharmacognosy, University of Graz, 8010 Graz, Austria; \\ julia.solnier@uni-graz.at \\ 2 Institute of Structural and Molecular Biology, Department of Biological Sciences, Birkbeck, University of \\ London, London WC1E 7HX, UK; liam.tom.martin@gmail.com (L.M.); s.bhakta@bbk.ac.uk (S.B.) \\ * Correspondence: franz.bucar@uni-graz.at; Tel.: +43-(316)-380-5531
}

Received: 20 December 2019; Accepted: 6 February 2020; Published: 7 February 2020

\begin{abstract}
Therapeutic treatment options for opportunistic non-tuberculous mycobacterial (NTM) infection and/or serious mycobacterial infections such as tuberculosis (TB) and leprosy are limited due to the spread of antimicrobial resistance mechanism. Plant-derived natural compounds as prospective efflux pump inhibitors may present a promising adjunct to conventional chemotherapy by enhancing mycobacterial susceptibility to antibiotics. This study served to evaluate the antimicrobial and resistance-modifying profile of a range of plant-derived flavonoids against the mycobacterial model strains: $M$. smegmatis, $M$. aurum, and $M$. bovis BCG. The minimum inhibitory concentrations (MICs) of the compounds against the mycobacterial strains were determined using both agar dilution and broth dilution assays, while their efflux inhibitory activity was investigated via an ethidium bromide-based fluorometric assay. All compounds were screened for their synergistic effects with ethidium bromide (EtBr) and rifampicin (RIF) against M. smegmatis. Skullcapflavone II (5,2'-dihydroxy-6,7,8,6' -tetramethoxyflavone, 1 ) exerted potent antimicrobial activity against $M$. aurum and $M$. bovis BCG and considerably increased the susceptibility of M. smegmatis to EtBr and RIF. Nobiletin $\left(5,6,7,8,3^{\prime}, 4^{\prime}\right.$-hexamethoxyflavone, 2$)$ was determined to be the most potent efflux-inhibitor in $M$. aurum and M. smegmatis. However, a connection between strong modulatory and putative efflux activity of the compounds could not be observed. Nevertheless, the results highlight two polymethoxyflavones, skullcapflavone II and nobiletin, with potent antimycobacterial and antibiotic resistance modulating activities as valuable adjuvants in anti-mycobacterial therapies.
\end{abstract}

Keywords: flavonoids; skullcapflavone II; nobiletin; efflux pumps; efflux pump inhibitors; mycobacteria

\section{Introduction}

The rapid global expansion of multidrug-resistant (MDR) and extensively drug-resistant (XDR) bacteria reflects the urgent need of a novel course in antibiotic therapy to tackle global infectious diseases such as tuberculosis (TB) [1].

According to the most recent Global Tuberculosis Report from the World Health Organization (WHO), TB remains one of the top 10 causes of death among infectious diseases. Due to the rising number of MDR strains, especially in mycobacteria including the Mycobacterium tuberculosis complex, as well as fast growing non-tuberculous strains, antimicrobial resistance has become a critical global health concern [2].

The Mycobacterium bovis bacillus Calmette-Guérin (BCG) strain is the most frequently used live attenuated vaccine against tuberculosis disease. The BCG strain was originally derived after 
several subcultures from its virulent progenitor Mycobacterium bovis (M. bovis), which triggers TB infections in animal species, predominantly cattle [3,4]. Beside the pathogenic mycobacteria, there are numerous opportunistic species occurring as saprophytes and commensals in the environment. These fast-growing and non-obligatory pathogenic organisms are categorized as non-tuberculous mycobacteria (NTM) that readily cause opportunistic infections in immunocompromised patients [5,6]. Among them, Mycobacterium smegmatis and Mycobacterium aurum regularly serve as low-pathogenic and rapidly growing surrogate models in antitubercular drug screening for antitubercular drugs $[7,8]$. Due to their genomic similarities and the correlation between their antibiotic susceptibility profile and that of M. tuberculosis, the use of model strains [9] including M. smegmatis, M. aurum, and M. bovis BCG accelerates the discovery of new antitubercular drugs, while lowering the risk to researchers, and allowing for screening of compounds in labs that lack Category 3 bio-safety facilities [6,10].

A distinctive feature of mycobacteria is their highly hydrophobic cell envelope and the prevalence of multidrug efflux pumps (EPs). Putative drug efflux genes and homologous pumps can be found in M. smegmatis, M. aurum, M. bovis, and M. tuberculosis [11-13]. These EPs represent one of many important resistance mechanisms developed by bacteria to survive in the presence of chemotherapeutic drugs [14]. By expelling toxic substrates from the bacterial cell, these transmembrane proteins operate as effective tools in order to prevent the intracellular accumulation of antimicrobial drugs $[15,16]$. Consequently, the inhibition of efflux pumps may be an effective strategy to assist in the fight against rising antibiotic resistance while initiating a new procedure in drug-therapy $[17,18]$.

Despite the fact that a number of challenges has to be overcome, like the risk of resistance development when mycobacteria are exposed to subinhibitory concentrations of potential efflux pump inhibitors (EPI), similar pharmacokinetics of adjuvant and antitubercular drugs or selectivity of EPI for bacterial efflux pumps rather than eukaryotic transporters, the inclusion of an EPI as part of a therapeutic regimen could revive the therapeutic efficacy of the fading antibiotic arsenal [10]. However, to date, no efflux pump inhibitor has been clinically approved [19]. Recently, interest has been growing in the identification of new efflux pump inhibitors from natural sources [20], including flavonoids. A number of flavonoids have been shown to increase susceptibility of NTM to isoniazid, the flavonol myricetin being the most active [21]. Further, the isoflavone biochanin A exhibited efflux pump inhibiting activity in M. smegmatis comparable to that of verapamil (VP) [22] and hence was used as template for synthesis of potent 3-phenylquinolone efflux inhibitors in M. avium [23]. Given the crucial problems posed by multidrug resistant pathogens, especially by mycobacteria, the administration of a plant-derived efflux pump inhibitor combined with an antibiotic may provide greater clinical benefits in the treatment of infectious diseases [24]. Flavonoids proved to be a promising group of plant phenolics in that respect and was hence investigated further in the present study by selecting more lipophilic structures, i.e., methoxylated derivatives (skullcapflavone II (1), nobiletin (2), tangeretin (3), wogonin (5)) and flavones lacking substituents at the C-2 aryl ring (baicalein (4), wogonin (5)) which might have a higher affinity for the lipid-rich mycobacterial cell envelope. Structures of the selected compounds can be depicted from Figure 1.

In this study, M. smegmatis, M. aurum, and M. bovis BCG were used as surrogate models for M. tuberculosis organism to analyze efflux-mediated resistance. We propose specific plant phenolic compounds, i.e., flavonoids, with strong antimycobacterial and resistance-modifying properties as valuable agents in the antibiotic therapy of mycobacterial infections. Additionally, we have demonstrated the ability of these phyto-compounds to impair the function of efflux pumps in mycobacteria. Two reference inhibitors, VP and chlorpromazine (CPZ), served to verify the efflux inhibiting profile of the suggested natural product compounds in the mycobacterial model strains. 
<smiles>COc1cccc(O)c1-c1cc(=O)c2c(O)c(OC)c(OC)c(OC)c2o1</smiles>

(1)<smiles>COc1ccc(-c2cc(=O)c3c(OC)c(OC)c(OC)c(OC)c3o2)cc1OC</smiles>

(2)<smiles>COc1ccc(-c2cc(=O)c3c(OC)c(OC)c(OC)c(OC)c3o2)cc1</smiles>

(3)<smiles>O=c1cc(-c2ccccc2)oc2cc(O)c(O)c(O)c12</smiles>

(4)<smiles>COc1c(O)cc(O)c2c(=O)cc(-c3ccccc3)oc12</smiles>

(5)

Figure 1. Chemical structures of skullcapflavone II (1), nobiletin (2), tangeretin (3), baicalein (4), and wogonin (5).

\section{Results}

\subsection{Antibacterial Activity}

Five plant-derived flavonoids, skullcapflavone II (1), nobiletin (2), tangeretin (3), baicalein (4), and wogonin (5) were assessed for their antimicrobial activities against the non-pathogenic model organisms M. smegmatis $\mathrm{mc}^{2} 155$, M. aurum, and M. bovis BCG. The minimum inhibitory concentrations (MICs) of the compounds as well as INH as reference antituberculotic against the studied strains are summarized in Table 1.

Briefly, the high-throughput spot culture growth inhibition (HT-SPOTi) assay [25,26] a rapid, gold standard, whole-cell screening method, was applied to investigate the anti-mycobacterial potential of the plant substrates against $M$. aurum and M. bovis BCG. The results indicate that all flavonoids exhibited strong antibacterial activities ( $\mathrm{MIC}_{99} \leq 31.25 \mathrm{mg} / \mathrm{L}$ ) against M. aurum. Skullcapflavone II (1) was the most potent compound, with an $\mathrm{MIC}_{99}$ of $7.8 \mathrm{mg} / \mathrm{L}$. However, baicalein (4) was the only compound showing strong antimycobacterial activity against M. smegmatis at a MIC99 of $32 \mathrm{mg} / \mathrm{L}$.

Table 1. Minimum inhibitory concentrations (MICs) of ethidium bromide and efflux pump inhibitors (EPIs) determined for M. smegmatis $\mathrm{mc}^{2} 155$ using microbroth dilution assay and spot culture growth inhibition (SPOTi)-assay for M. aurum and M. bovis BCG.

\begin{tabular}{cccc}
\hline & \multicolumn{3}{c}{ MIC $_{\mathbf{9 9}}$ (mg/L) } \\
\cline { 2 - 4 } Compound & M. smegmatis $\mathbf{~ m c ~}^{\mathbf{2}} \mathbf{~ 1 5 5}$ & M. aurum ATCC 23366 & M. bovis BCG ATCC 35734 \\
\hline Skullcapflavone II (1) & 128 & 7.8 & 31.25 \\
Nobiletin (2) & 128 & 31.25 & 62.5 \\
Tangeretin (3) & 128 & 31.25 & 31.25 \\
Baicalein (4) & 32 & 31.25 & 250 \\
Wogonin (5) & 128 & 31.25 & 500 \\
Isoniazid (INH) & 4 & 0.5 & 0.1 \\
Verapamil (VP) & 250 & 250 & $320[27]$ \\
Chlorpromazine (CPZ) & 25 & 20 & $20[27]$ \\
Ethidium bromide (EtBr) & 6.25 & 1 & $0.5[27]$ \\
\hline
\end{tabular}

\subsection{Resistance Modulatory Activity}

The resistance-modulating profile of the compounds towards $M$. smegmatis, a model strain that expresses a range of efflux pumps, was determined using a microtiter broth dilution assay. All compounds were tested at sub-inhibitory concentrations correlating to one-quarter of their MIC in 
M. smegmatis. The modulation factor (MF) indicates the resistance-modifying impact of the compounds on the MICs of ethidium bromide (EtBr) and rifampicin (RIF), a frontline antitubercular drug. Previous resistance modulation experiments in $M$. smegmatis with RIF showed better correlation to EtBr modulation than with INH [28], hence in the current study, RIF was preferred. Rodriguez et al. [29] tested RIF in combination with VP and CPZ in M. smegmatis mc $^{2} 155$ wild-type strain, which revealed a MF of 2 (VP) and 4 (CPZ), respectively. This also supported our selection of the M. smegmatis model and RIF as anti-tuberculotic drug in order to assay the flavonoids 1-5 for potential resistance modifying effects related to efflux pump inhibition. Although RIF resistance in $M$. tuberculosis strains mainly refers to chromosomal mutations, the overexpression of efflux pumps in a higher number of RIF-resistant strains compared to RIF-sensitive strains points towards a contribution of efflux to RIF resistance of M. tuberculosis [30].

Given the results of the modulatory screening presented in Table 2, it can be observed that the majority of the compounds affected the susceptibility of M. smegmatis towards EtBr and RIF, albeit to varying extents. Of all compounds tested, compound 1 proved to be the most effective modulator, causing a remarkable increase in the susceptibility of $M$. smegmatis to $\mathrm{EtBr}(\mathrm{MF}=128)$ and enhanced the activity of the anti-TB drug RIF to 4-fold.

Table 2. Determination of the MICs and modulation factors of the compounds for M. smegmatis mc ${ }^{2}$ 155 by using microbroth dilution assays.

\begin{tabular}{ccccc}
\hline Compound & MIC (mg/L) & $\begin{array}{c}\text { [c] as Modulator } \\
(\mathbf{m g} / \mathbf{L})\end{array}$ & $\begin{array}{c}\text { MIC Mod. } \\
(\mathbf{m g} / \mathbf{L}) / \mathbf{M F} \text { EtBr }\end{array}$ & $\begin{array}{c}\text { MIC Mod. } \\
(\mathbf{m g} / \mathbf{L}) / \mathbf{M F} \text { RIF }\end{array}$ \\
\hline Skullcapflavone II (1) & 128 & 32 & $0.0625 / 128$ & $8 / 4$ \\
Nobiletin (2) & 128 & 32 & $2 / 4$ & $32 / 1$ \\
Tangeretin (3) & 128 & 32 & $2 / 4$ & $32 / 1$ \\
Baicalein (4) & 32 & 8 & $8 / 1$ & $32 / 1$ \\
Wogonin (5) & 128 & 32 & $4 / 2$ & $16 / 2$ \\
\hline
\end{tabular}

Minimum inhibitory concentration (MIC) of ethidium bromide $(\mathrm{EtBr})=8 \mathrm{mg} / \mathrm{L}$ and MIC of rifampicin $(\mathrm{RIF})=32 \mathrm{mg} / \mathrm{L}$. Modulation factor $(\mathrm{MF})=\mathrm{MIC}(\mathrm{EtBr}$ or RIF)/MIC (EtBr or RIF + modulator); $n=4$. MIC mod. $=$ MIC of EtBr or RIF in presence of the modulator at a concentration corresponding to a quarter of its MIC.

\subsection{EtBr Accumulation}

Ethidium bromide, a known substrate of efflux pumps, is commonly used to assess efflux activity due to its ability to fluoresce when binding to hydrophobic regions within the bacterial cell [26]. The increase of EtBr-accumulation in the presence of a potential EPI indicates an interplay between cell-wall permeability and inhibition of efflux activity, which can be detected by fluorometric measurement $[10,31]$.

Therefore, in order to assess the potential of the flavonoid compound group to inhibit mycobacterial efflux pumps, EtBr-accumulation assays were performed using the known EPIs VP and CPZ as reference inhibitors. Compounds were evaluated for their capability to accumulate EtBr in M. smegmatis and M. aurum. As shown in a previous study of Rodriguez et al. [27] in M. bovis BCG, only a basal efflux activity with EtBr took place, and the effects of EPIs in this strain were less clearly observable. This is also in agreement with our observations (unpublished data). Hence, in this study, we preferred to use M. smegmatis and M. aurum as model strains for EtBr accumulation in mycobacteria. Verapamil, a calcium-channel antagonist, clinically used for the treatment of cardiac disorders, is a well-known inhibitor of p-glycoprotein in mammalian cells [32] and was also found to inhibit ATP-dependent multidrug transporters (ABC pumps) in prokaryotic cells such as the DrrAB pump of M. tuberculosis [33, 34]. VP and phenothiazines like CPZ are regarded as compounds, which interfere directly or indirectly with the proton motive force, the energy source of many secondary transporters [27].

Compounds were tested at concentrations half of their MIC together with $0.4 \%$ glucose and EtBr at $0.5 \mathrm{mg} / \mathrm{L}$, the lowest concentration of EtBr that resulted in minimal accumulation by M. smegmatis 
and M. aurum within $60 \mathrm{~min}$ at $37^{\circ} \mathrm{C}$. The reference inhibitor VP indicated the highest level of EtBr-accumulation in both mycobacterial strains, while CPZ was less effective.

In EtBr accumulation assays with $M$. smegmatis, only flavonoids 2 and $\mathbf{4}$ caused an increase in accumulation similar to CPZ. As Figure 2a demonstrates, all other compounds could slightly potentiate accumulation when compared to the negative control but were less effective than the reference inhibitors.

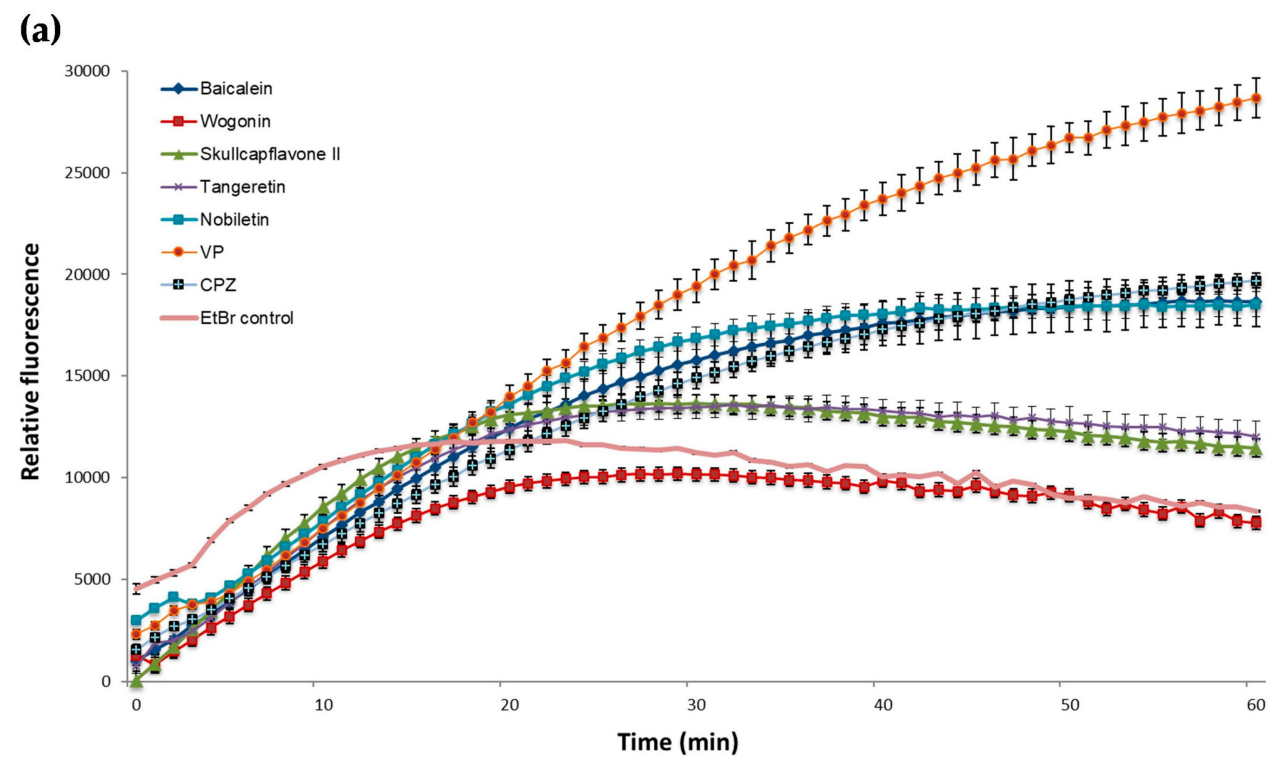

(b)

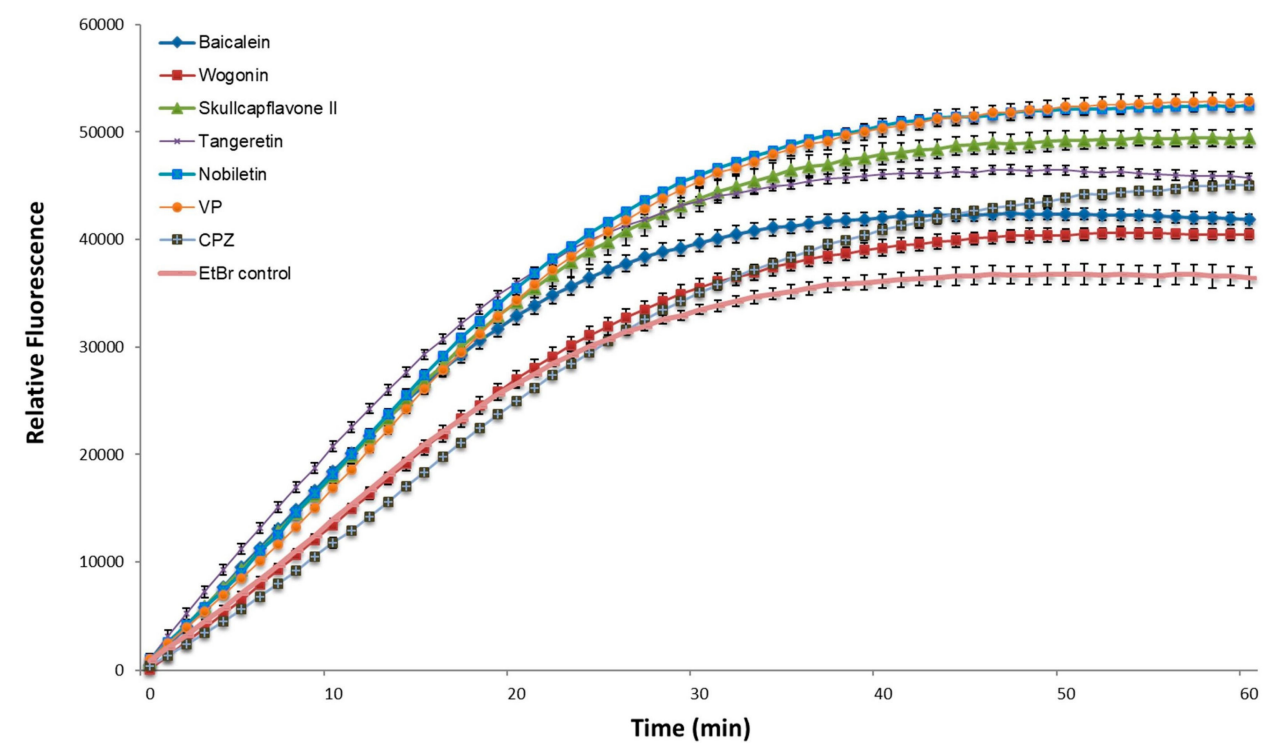

Figure 2. Effect of the potential EPIs and reference inhibitors on the EtBr accumulation in M. smegmatis $\mathrm{mc}^{2} 155$ (a) and M. aurum ATCC 23366 (b). All compounds were tested at sub inhibitory concentrations at half of their MIC (Table 1). EtBr, ethidium bromide was used as negative control (drug-free culture) at a final concentration of $0.5 \mathrm{mg} / \mathrm{L}$. VP, verapamil, a well-known efflux pump inhibitor and CPZ, chlorpromazine, were integrated as positive controls. The experiments were performed in triplicate $(n=3)$; values represent means $+\mathrm{SD}$.

Compound 2 was shown to be the most effective promoter for EtBr accumulation in M. aurum (Figure 2b), with a similar efficacy to that of VP. Compounds $\mathbf{1}$ and $\mathbf{3}$ enhanced EtBr-accumulation in M. aurum to a greater extent than CPZ but were less effective than VP. Flavonoids 4 and 5 were the least effective of the compounds tested against $M$. aurum. 


\section{Discussion}

A small chemical series of plant-derived flavonoid compounds (1-5) was screened for antimycobacterial activity against a range of fast- and slow-growing mycobacterial model organisms. Efflux-pump inhibition and modulatory activity were tested against the fast-growing mycobacterial model organisms $M$. smegmatis and $M$. aurum, due to the limitations of the assay with regards to the slow-growing nature of $M$. bovis BCG.

Considering the structural similarities of the tested flavonoids, particular structural moieties in the molecule appear to be crucial for the antimicrobial properties. The antibacterial activity of flavonoids may be related to structural features such as the number and positions of methoxy and hydroxyl groups [35,36]. The 5,6,7-trihydroxy substitution pattern of compound 4 appears to enhance the antimycobacterial effect against $M$. smegmatis, as can be observed by comparison with the structurally similar flavonoid 5. It is notable that the most potent inhibitor of M. smegmatis is the most hydrophilic of the five flavonoids tested. This may be influenced by the fact that, unlike the other mycobacterial strains, M. smegmatis possesses a higher number of porin-like channels such as the major porin MspA, which allows the diffusion of hydrophilic compounds through the hydrophobic bilayer of the cell wall $[37,38]$. On the other hand, the only flavonoid with the unusual $2^{\prime}, 6^{\prime}$-substitution pattern, i.e., compound 1, was the most active in $M$. aurum, and the more lipophilic polymethoxylated flavonoids 1-3 showed lower MIC values in $M$. bovis BCG compared to those compounds with no substituents at the C-2 aryl ring (compounds 4,5 ), Table 1 . In a previous study, it was reported that the presence of a lipophilic group at position 6 or 8 as well as hydroxylation at position 5 such as in compound 1 improves the antibacterial activity of flavonoids [39].

It is notable that across the three model organisms used in this study, the MIC 99 values and relative potency of the five flavonoids shows significant variation. Such differences have been reported in other publications and may result from any of a number of phenotypic and genotypic variations between the three species $[6,40]$. For example, the number of unique proteins found in M. smegmatis was 224 greater than for M. aurum, M. bovis BCG, and M. tuberculosis [6].

Skullcapflavone II (1) was also identified as the most potent modulator of the antibacterial susceptibility of the compounds studied in M. smegmatis, with a MF of 128 when combined with EtBr and a MF of 4 when combined with RIF. It is important to note however, the results of the modulation assays simply indicate a possible trend towards efflux inhibition rather than a precise prediction [41,42]. An important role of efflux pumps, specifically LfrA, in resistance to M. smegmatis to RIF can be deduced from the results of Rodriguez et al. [29] who found a significant reduction of MIC for RIF when comparing susceptibility of wild-type strain (MIC $=4 \mathrm{mg} / \mathrm{L}$ ) and a lfrA gene knockout strain (MIC $=0.5 \mathrm{mg} / \mathrm{L}$ ) of $M$. smegmatis $\mathrm{mc}^{2} 155$. However, in the current study, no specific mycobacterial efflux pump has been focused. Comparison of compound $\mathbf{1}$ and the similar flavonoids $\mathbf{2}$ and 3 suggests that the replacement of the C-5 methoxy group with a hydroxyl group may be significant. However, the substitution on the C-2 aryl ring may also have a significant influence of their EtBr- and RIF-modulating abilities. However, compounds 2 and 3, both polymethoxyflavones distinguished by one methoxy- group at C-3', exhibited similar modulating effects against the tested strains.

All compounds were observed to increase the level of EtBr-accumulation in M. smegmatis (with the exception of 5) and M. aurum in relation to the negative control. Compound 2 in particular displayed potent efflux-inhibitory activity, and by comparison with the closely related flavonoid 3 , it can be observed that the additional $3^{\prime}$-methoxy group significantly improved the efflux inhibitory activity of this scaffold. Interestingly, flavonoid 4 caused a significant accumulation level for EtBr in M. smegmatis comparable to the effect of CPZ but had no modulatory activity on EtBr $(\mathrm{MF}=1)$. On the other hand, compound 1, which appeared to be a highly efficient modulator for EtBr against M. smegmatis $(\mathrm{MF}=128)$, was comparatively less potent in terms of efflux inhibition. Consequently, a connection between strong modulatory and putative efflux activity of the compounds could not be observed. It has to be taken into consideration that due to the different exposure times of the bacteria to the test compounds and EtBr, i.e., the test substrate of potential efflux pumps, a comparison between 
both types of assay cannot readily be established. In the case of the modulation assay, incubation for $72 \mathrm{~h}$ takes place, whereas in the case of the accumulation assay, the mycobacteria are exposed for only $1 \mathrm{~h}$ to the respective chemicals. In addition, a high modulating activity in combination with $\mathrm{EtBr}$, as in case of compound 1, might influence the viability of the mycobacteria in the accumulation assay and finally lead to a less pronounced $\mathrm{EtBr}$ accumulation effect as one might expect from results of modulation assay. In previous experiments, we could show that VP and CPZ have a relatively low MF when combined with $\mathrm{EtBr}$ in $M$. smegmatis (VP, MF $=2$ at $40 \mathrm{mg} / \mathrm{L} ; \mathrm{CPZ}, \mathrm{MF}=2$ at 32 $\mathrm{mg} / \mathrm{L}$ ) [22], however both compounds clearly facilitated EtBr accumulation in the fluorescence-based accumulation assay. Cell wall impermeability in conjunction with the individual numbers of efflux pumps present in each mycobacterial strain may contribute to the diversity of drug susceptibility between the model strains. The overactivity of efflux systems as well as the mode of inhibition is still mostly unexplored [43].

The results of this study can be taken as a starting point for more in depth investigation of skullcapflavone II (1) and nobiletin (2) as adjuvants in antimycobacterial combination therapy. Several polymethoxylated flavones, bearing four or more methoxyl groups on their benzo- $\gamma$-pyrone skeleton, have previously been shown to possess a broad spectrum of biological activities, including anti-inflammatory, anti-carcinogenic, antiviral, antioxidant, anti-thrombogenic, and anti-atherogenic properties [39,44].

\section{Materials and Methods}

\subsection{Drugs and Inhibitors}

Plant compounds skullcapflavone II (1), nobiletin (2), and tangeretin (3) were purchased from ChemFaces Biochemical Co., Wuhan, China. Baicalein (4) and wogonin (5) were obtained from Sigma Aldrich. Purities of the compounds were $\geq 98 \%$. Chlorpromazine, verapamil, rifampicin, ethidium bromide, isoniazid, and phosphate-buffered saline (PBS) in tablets $(0.01 \mathrm{M}$ phosphate buffer, $0.0027 \mathrm{M}$ $\mathrm{KCl}, 0.14 \mathrm{M} \mathrm{NaCl}$ and 0,05\% Tween 80, $\mathrm{pH}=7.4$ ) were purchased from Sigma Aldrich (Poole, UK).

All compounds were dissolved either in dimethyl sulfoxide (DMSO) or in sterile deionized water.

\subsection{Bacterial Strains and Growth Media}

The bacterial strains Mycobacterium smegmatis $\mathrm{mc}^{2} 155$ ATCC 700084, Mycobacterium aurum ATCC 23366, and Mycobacterium bovis BCG ATCC 35734 were cultivated either on Columbia blood agar supplemented with 5\% defibrinated horse blood (MIC and modulation assay) or in Middlebrook 7H9 broth supplemented with $10 \%$ of OADC enrichment (SPOTi and ethidium bromide accumulation assay) at $37^{\circ} \mathrm{C}$ under aerobic conditions. Bacterial strains were purchased from UK National Collection of Type Cultures (NCTC). Middlebrook 7H9 broth supplemented with Middlebrook OADC Enrichment and $0.05 \%$ Tween 80 (Sigma-Aldrich) or $0.4 \%$ glycerol used for efflux assays were purchased from Becton-Dickinson (Oxford, UK). Mueller-Hinton Broth (cation-adjusted) was used for MIC and modulation experiments.

\subsection{Drug Susceptibility Testing}

Minimum inhibitory concentrations (MIC) of the compounds against non-pathogenic strains Mycobacterium aurum and Mycobacterium bovis BCG were determined by using the spot-culture growth inhibition assay (SPOTi) according to Evangelopoulos and Bhakta [25].

Isoniazid, a first-line antitubercular drug, was included as a positive control. All experiments were performed in triplicate. The MIC, defined as the lowest concentration of a given compound required to inhibit bacterial growth, was visually determined.

Microbroth dilution assays served to investigate the antimicrobial and resistance-modulating effects of the compounds against Mycobacterium smegmatis mc $^{2} 155$ (ATCC 700084) and were conducted as described in literature in accordance with NCCLS guidelines [43]. 
Briefly, all compounds were dissolved in dimethyl sulfoxide (DMSO) and diluted in Mueller-Hinton broth before testing in 96-well microtiter plates. The concentration of DMSO did not exceed $3.6 \%$ for the final assay. For each plate, an additional sterile and growth control as well as a positive control (Isoniazid) was included. The plates were inoculated with a $5 \times 10^{5}$ colony forming units $(\mathrm{CFU}) / \mathrm{mL}$ bacterial suspension, adjusted to equal the McFarland 0.5 turbidity standard, and were incubated at $37^{\circ} \mathrm{C}$ for $72 \mathrm{~h}$. For the assessment of cell viability, the determination of the MIC was carried out using MTT (3-(4,5-dimethylthiazol-2-yl)-2,5-diphenyltetrazolium bromide (Sigma-Aldrich), indicating bacterial growth by a visible color change from yellow to blue [45].

\subsection{Modulation Factor Analysis}

Additionally, test compounds were further analyzed for their modulatory activity on the MIC of ethidium bromide (EtBr) and rifampicin (RIF), respectively.

At sub-inhibitory concentrations corresponding to one-quarter of their MIC, the concentration of the compounds was kept constant while EtBr and RIF were serially diluted. The modulation factor (MF) reflected the modulating impact of the compounds on the MIC of the antibiotic and ethidium bromide, calculated according to the formula:

$$
\mathrm{MF}=(\mathrm{MIC} \text { antibiotic }) /(\mathrm{MIC} \text { antibiotic }+ \text { modulator })
$$

\subsection{Whole Cell Efflux Assay}

EtBr accumulation assay for the bacterial strains Mycobacterium smegmatis $m c^{2} 155$ and Mycobacterium aurum was adapted following the procedure of Rodrigues et al. [7]. Each compound was tested at sub inhibitory concentrations correlating to one-half of their MIC.

Bacterial cultures were grown in $10 \mathrm{~mL}$ of Middlebrook 7H9 broth medium supplemented with $\mathrm{OADC}$ at $37^{\circ} \mathrm{C}$ until an $\mathrm{OD}_{600}$ of 0.8 was reached. After adjusting the $\mathrm{OD}_{600}$ to 0.4 in Middlebrook $7 \mathrm{H} 9$ broth, the bacterial suspension was centrifuged at $3000 \mathrm{rpm}$ for $10 \mathrm{~min}$. The supernatant was discarded, and the pellet re-suspended in $10 \mathrm{~mL}$ of PBS containing $0.05 \%$ Tween 80 . Test compounds at sub-inhibitory concentrations (MIC $1 / 2$ ) together with glucose (final concentration of $0.4 \%$ ) were prepared in microtubes containing $0.5 \mathrm{~mL}$ of bacterial suspension. Aliquots of $0.1 \mathrm{~mL}$ of each working solution were transferred into separate wells of a 96-well plate. Ethidium bromide was added to each well to a final concentration of $0.5 \mathrm{mg} / \mathrm{L}$.

The 96-well plate was placed in a fluorimeter (FLUOstar OPTIMA, BMG Labtech) and fluorescence data were recorded every $60 \mathrm{~s}$ for $60 \mathrm{~min}$ at $37^{\circ} \mathrm{C}$ using an excitation wavelength of $544 \mathrm{~nm}$ and an emission wavelength of $590 \mathrm{~nm}$. The effect of the reference inhibitors VP and CPZ on the accumulation of $\mathrm{EtBr}$ at $37^{\circ} \mathrm{C}$ was determined as described before. All experiments were performed in triplicate.

In order to evaluate the homogeneity of variance and statistical significance of the efflux results, the f-test and two-tailed t-test were conducted to allow comparison of the test compounds to the EtBr control (Table 3). 
Table 3. Results of the relative fluorescence of accumulation.

\begin{tabular}{ccccc}
\hline & \multicolumn{2}{c}{ M. smegmatis $\mathbf{m c}^{\mathbf{2}} \mathbf{1 5 5}$} & \multicolumn{2}{c}{ M. aurum ATCC 23366 } \\
\cline { 2 - 5 } Compound & Mean Value & $\begin{array}{c}\text { Standard } \\
\text { Deviation }\end{array}$ & Mean Value & $\begin{array}{c}\text { Standard } \\
\text { Deviation }\end{array}$ \\
\hline Baicalein & $18573,3^{* * *}$ & 102,1 & $42146,5^{* * *}$ & 151,3 \\
Wogonin & $8376,9^{* *}$ & 326,1 & $40517,0^{* * *}$ & 63,1 \\
Skullcapflavone II & $11811,1^{* * *}$ & 239,6 & $49361,2^{* * *}$ & 82,5 \\
Tangeretin & $12400,6^{* * *}$ & 202,7 & $46077,8^{* * *}$ & 213,4 \\
Nobiletin & $18451,2^{* * *}$ & 33,8 & $52316,3^{* * *}$ & 117,2 \\
EtBr control & 8778,6 & 223,8 & 36683,7 & 93,2 \\
& $n=60$ & & $n=60$ &
\end{tabular}

Calculated as measured during the last $10 \mathrm{~min}$ of the assays. Compounds were compared to the EtBr control. Asterisks indicate the level of significance: ${ }^{* * *} p<0.001,{ }^{* *} p<0.01$.

\section{Conclusions}

The polymethoxylated flavonoids skullcapflavone II (1) and nobiletin (2) were found to be potent inhibitors of the growth of M. aurum and M. bovis BCG and interfered with efflux pump activity in M. aurum and M. smegmatis. In particular, flavonoid 1 exerted a remarkable modulating impact on $\mathrm{EtBr}$ and significantly decreased the rifampicin-resistance level of $M$. smegmatis. These results are significant as several reports have shown that efflux pump inhibitors are able to inhibit the survival of intracellular M. tuberculosis and potentiate the activity of anti-TB drugs [46,47]. However, it was not possible to observe a clear connection between strong modulatory and putative efflux activity of the compounds.

To date, there have been no published studies into the antimycobacterial efficacy of skullcapflavone II (1) and nobiletin (2) or their inhibitory activity on drug efflux in mycobacteria. It is imperative that we explore new strategies in TB treatment and management, and therefore we propose these flavonoids are valuable candidates for the inhibition of mycobacterial efflux pumps and the improvement of antibiotic performance against tuberculosis and other non-tubercular mycobacterial infections.

Author Contributions: J.S. performed the MIC, SPOTi, and Modulation assays as well as the Accumulation assays and drafted the manuscript-original draft preparation; L.M. assisted and helped to perform the SPOTi and Accumulation assays and drafted the manuscript-review and editing; S.B. supervised the experiments such as SPOTi assays and Accumulation assays conducted with M. smegmatis, M. aurum, and M. bovis BCG and contributed to drafting the manuscript; F.B. supervised the experiments such as MIC and Modulation assays performed on M. smegmatis, helped to analyze and interpret data, and contributed to drafting the manuscript-review and editing. All authors have read and agreed to the published version of the manuscript.

Funding: The authors acknowledge financial support by the University of Graz awarding a short-term mobility (KUWI) fellowship for J.S. and a Wellcome Trust doctoral training scholarship to L.M. at the Institute of Structural and Molecular Biology. Open Access Funding by the University of Graz.

Acknowledgments: We would like to thank Arundhati Maitra of the Institute of Structural and Molecular Biology, Department of Biological Sciences at Birkbeck, University London, for helpful discussions.

Conflicts of Interest: The authors declare no conflict of interest. The funders had no role in the design of the study; in the collection, analyses, or interpretation of data; in the writing of the manuscript, or in the decision to publish the results.

\section{References}

1. Guzman, J.D.; Evangelopoulos, D.; Gupta, A.; Birchall, K.; Mwaigwisya, S.; Saxty, B.; McHugh, T.D.; Gibbons, S.; Malkinson, J.; Bhakta, S. Antitubercular specific activity of ibuprofen and the other 2-arylpropanoic acids using the HT-SPOTi whole-cell phenotypic assay. BMJ Open 2013. [CrossRef] [PubMed]

2. World Health Organization (WHO). Global Tuberculosis Report 2019. Geneva: World Health Organization; 2019. Licence: CC BY-NC-SA 3.0 IGO. Available online: https:/apps.who.int/iris/bitstream/handle/10665/ 329368/9789241565714-eng.pdf?ua=1 (accessed on 23 December 2019). 
3. Mahairas, G.G.; Sabo, P.J.; Hickey, M.J.; Singh, D.C.; Stover, C.K. Molecular analysis of genetic differences between Mycobacterium bovis BCG and virulent M. bovis. J. Bacteriol. 1996, 178, 1274-1282. [CrossRef] [PubMed]

4. Ritz, N.; Tebruegge, M.; Connell, T.G.; Sievers, A.; Robins-Browne, R.; Curtis, N. Susceptibility of Mycobacterium bovis BCG vaccine strains to antituberculous antibiotics. Antimicrob. Agents Chemother. 2009, 53, 316-318. [CrossRef]

5. $\quad$ van Ingen, J.; Boeree, M.J.; van Soolingen, D.; Mouton, J.W. Resistance mechanisms and drug susceptibility testing of nontuberculous mycobacteria. Drug Resist. Updat. 2012, 15, 149-161. [CrossRef] [PubMed]

6. Phelan, J.; Maitra, A.; McNerney, R.; Nair, M.; Gupta, A.; Coll, F.; Pain, A.; Bhakta, S.; Clark, T.G. The draft genome of Mycobacterium aurum, a potential model organism for investigating drugs against Mycobacterium tuberculosis and Mycobacterium leprae. Int. J. Mycobacteriol. 2015, 4, 207-216. [CrossRef]

7. Rodrigues, L.; Wagner, D.; Viveiros, M.; Sampaio, D.; Couto, I.; Vavra, M.; Kern, W.V.; Amaral, L. Thioridazine and chlorpromazine inhibition of ethidium bromide efflux in Mycobacterium avium and Mycobacterium smegmatis. J. Antimicrob. Chemother. 2008, 61, 1076-1082. [CrossRef]

8. Sood, S.; Yadav, A.; Shrivastava, R. Mycobacterium aurum is unable to survive Mycobacterium tuberculosis latency associated stress conditions: Implications as non-suitable model organism. Indian J. Microbiol. 2016, 56, 198-204. [CrossRef]

9. Altaf, M.; Miller, C.H.; Bellows, D.S.; O'Toole, R. Evaluation of the Mycobacterium smegmatis and BCG models for the discovery of Mycobacterium tuberculosis inhibitors. Tuberculosis 2010, 90, 333-337. [CrossRef]

10. Stavri, M.; Piddock, L.J.V.; Gibbons, S. Bacterial efflux pump inhibitors from natural sources. J. Antimicrob. Chemother. 2007, 59, 1247-1260. [CrossRef]

11. Li, X.-Z.; Zhang, L.; Nikaido, H. Efflux pump-mediated intrinsic drug resistance in Mycobacterium smegmatis. Antimicrob. Agents Chemother. 2004, 48, 2415-2423. [CrossRef]

12. Namouchi, A.; Cimino, M.; Favre-Rochex, S.; Charles, P.; Gicquel, B. Phenotypic and genomic comparison of Mycobacterium aurum and surrogate model species to Mycobacterium tuberculosis: Implications for drug discovery. BMC Genom. 2017. [CrossRef] [PubMed]

13. Silva, P.E.; Bigi, F.; Santangelo, M.P.; Romano, M.I.; Martín, C.; Cataldi, A.; Aínsa, J.A. Characterization of P55, a multidrug efflux pump in Mycobacterium bovis and Mycobacterium tuberculosis. Antimicrob. Agents Chemother. 2001, 45, 800-804. [CrossRef] [PubMed]

14. Fernández, L.; Hancock, R.E.W. Adaptive and mutational resistance: Role of porins and efflux pumps in drug resistance. Clin. Microbiol. Rev. 2012, 25, 661-681. [CrossRef] [PubMed]

15. Puzari, M.; Chetia, P. RND efflux pump mediated antibiotic resistance in Gram-negative bacteria Escherichia coli and Pseudomonas aeruginosa: A major issue worldwide. World J. Microbiol. Biotechnol. 2017. [CrossRef] [PubMed]

16. Mikulášová, M.; Chovanová, R.; Vaverková, Š. Synergism between antibiotics and plant extracts or essential oils with efflux pump inhibitory activity in coping with multidrug-resistant staphylococci. Phytochem. Rev. 2016, 15, 651-662. [CrossRef]

17. Viveiros, M.; Martins, M.; Rodrigues, L.; Machado, D.; Couto, I.; Ainsa, J.; Amaral, L. Inhibitors of mycobacterial efflux pumps as potential boosters for anti-tubercular drugs. Expert Rev. Anti Infect. Ther. 2012, 10, 983-998. [CrossRef]

18. Shriram, V.; Khare, T.; Bhagwat, R.; Shukla, R.; Kumar, V. Inhibiting bacterial drug efflux pumps via phyto-therapeutics to combat threatening antimicrobial resistance. Front. Microbiol. 2018. [CrossRef]

19. Alav, I.; Sutton, J.M.; Rahman, K.M. Role of bacterial efflux pumps in biofilm formation. J. Antimicrob. Chemother. 2018, 73, 2003-2020. [CrossRef]

20. Prasch, S.; Duran, A.G.; Chinchilla, N.; Molinillo, J.M.G.; Macías, F.A.; Bucar, F. Resistance modulatory and efflux-inhibitory activities of capsaicinoids and capsinoids. Bioorg. Chem. 2019, 82, 378-384. [CrossRef]

21. Lechner, D.; Gibbons, S.; Bucar, F. Modulation of isoniazid susceptibility by flavonoids in Mycobacterium. Phytochem. Lett. 2008, 1, 71-75. [CrossRef]

22. Lechner, D.; Gibbons, S.; Bucar, F. Plant phenolic compounds as ethidium bromide efflux inhibitors in Mycobacterium smegmatis. J. Antimicrob. Chemother. 2008, 62, 345-348. [CrossRef] [PubMed] 
23. Cannalire, R.; Machado, D.; Felicetti, T.; Santos Costa, S.; Massari, S.; Manfroni, G.; Barreca, M.L.; Tabarrini, O.; Couto, I.; Viveiros, M.; et al. Natural isoflavone biochanin A as a template for the design of new and potent 3-phenylquinolone efflux inhibitors against Mycobacterium avium. Eur. J. Med. Chem. 2017, 140, 321-330. [CrossRef] [PubMed]

24. Amaral, L.; Kristiansen, J.E.; Viveiros, M.; Atouguia, J. Activity of phenothiazines against antibiotic-resistant Mycobacterium tuberculosis: A review supporting further studies that may elucidate the potential use of thioridazine as anti-tuberculosis therapy. J. Antimicrob. Chemother. 2001, 47, 505-511. [CrossRef] [PubMed]

25. Evangelopoulos, D.; Bhakta, S. Rapid methods for testing inhibitors of mycobacterial growth. Methods Mol. Biol. 2010, 642, 193-201. [CrossRef]

26. Danquah, C.A.; Kakagianni, E.; Khondkar, P.; Maitra, A.; Rahman, M.; Evangelopoulos, D.; McHugh, T.D.; Stapleton, P.; Malkinson, J.; Bhakta, S.; et al. Analogues of disulfides from Allium stipitatum demonstrate potent anti-tubercular activities through drug efflux pump and biofilm inhibition. Sci. Rep. 2018. [CrossRef]

27. Rodrigues, L.; Machado, D.; Couto, I.; Amaral, L.; Viveiros, M. Contribution of efflux activity to isoniazid resistance in the Mycobacterium tuberculosis complex. Infect. Genet. Evol. 2012, 12, 695-700. [CrossRef]

28. Gröblacher, B.; Maier, V.; Kunert, O.; Bucar, F. Putative mycobacterial efflux inhibitors from the seeds of Aframomum melegueta. J. Nat. Prod. 2012, 75, 1393-1399. [CrossRef]

29. Rodrigues, L.; Ramos, J.; Couto, I.; Amaral, L.; Viveiros, M. Ethidium bromide transport across Mycobacterium smegmatis cell-wall: Correlation with antibiotic resistance. BMC Microbiol. 2011. [CrossRef]

30. Narang, A.; Garima, K.; Porwal, S.; Bhandekar, A.; Shrivastava, K.; Giri, A.; Sharma, N.K.; Bose, M.; Varma-Basil, M. Potential impact of efflux pump genes in mediating rifampicin resistance in clinical isolates of Mycobacterium tuberculosis from India. PLoS ONE 2019, 14, e0223163. [CrossRef]

31. Bansal, A.; Mallik, D.; Kar, D.; Ghosh, A.S. Identification of a multidrug efflux pump in Mycobacterium smegmatis. FEMS Microbiol. Lett. 2016. [CrossRef]

32. Pule, C.M.; Sampson, S.L.; Warren, R.M.; Black, P.A.; van Helden, P.D.; Victor, T.C.; Louw, G.E. Efflux pump inhibitors: Targeting mycobacterial efflux systems to enhance TB therapy. J. Antimicrob. Chemother. 2016, 71, 17-26. [CrossRef] [PubMed]

33. Pasca, M.R.; Guglierame, P.; Arcesi, F.; Bellinzoni, M.; De Rossi, E.; Riccardi, G. Rv2686c-Rv2687c-Rv2688c, an ABC fluoroquinolone efflux pump in Mycobacterium tuberculosis. Antimicrob. Agents Chemother. 2004, 48, 3175-3178. [CrossRef] [PubMed]

34. Rodrigues, L.; Ainsa, J.A.; Amaral, L.; Viveiros, M. Inhibition of Drug Efflux in Mycobacteria with Phenothiazines and Other Putative Efflux Inhibitors. PRI 2011, 6, 118-127. [CrossRef] [PubMed]

35. Alcaráz, L.E.; Blanco, S.E.; Puig, O.N.; Tomás, F.; Ferretti, F.H. Antibacterial activity of flavonoids against methicillin-resistant Staphylococcus aureus strains. J. Theor. Biol. 2000, 205, 231-240. [CrossRef]

36. Tsuchiya, H.; Sato, M.; Miyazaki, T.; Fujiwara, S.; Tanigaki, S.; Ohyama, M.; Tanaka, T.; Iinuma, M. Comparative study on the antibacterial activity of phytochemical flavanones against methicillin-resistant Staphylococcus aureus. J. Ethnopharmacol. 1996, 50, 27-34. [CrossRef]

37. Niederweis, M. Nutrient acquisition by mycobacteria. Microbiology 2008, 154, 679-692. [CrossRef] [PubMed]

38. Lambert, P.A. Cellular impermeability and uptake of biocides and antibiotics in Gram-positive bacteria and mycobacteria. J. Appl. Microbiol. 2002. [CrossRef]

39. Liu, L.; Xu, X.; Cheng, D.; Yao, X.; Pan, S. Structure-activity relationship of citrus polymethoxylated flavones and their inhibitory effects on Aspergillus niger. J. Agric. Food Chem. 2012, 60, 4336-4341. [CrossRef]

40. Mohan, A.; Padiadpu, J.; Baloni, P.; Chandra, N. Complete Genome Sequences of a Mycobacterium smegmatis Laboratory Strain (MC2 155) and Isoniazid-Resistant (4XR1/R2) Mutant Strains. Genome Announc. 2015. [CrossRef]

41. Sharma, S.; Kumar, M.; Sharma, S.; Nargotra, A.; Koul, S.; Khan, I.A. Piperine as an inhibitor of Rv1258c, a putative multidrug efflux pump of Mycobacterium tuberculosis. J. Antimicrob. Chemother. 2010, 65, 1694-1701. [CrossRef]

42. Zaw, M.T.; Emran, N.A.; Lin, Z. Mutations inside rifampicin-resistance determining region of rpoB gene associated with rifampicin-resistance in Mycobacterium tuberculosis. J. Infect. Public Health 2018, 11, 605-610. [CrossRef] [PubMed]

43. Gröblacher, B.; Kunert, O.; Bucar, F. Compounds of Alpinia katsumadai as potential efflux inhibitors in Mycobacterium smegmatis. Bioorg. Med. Chem. 2012, 20, 2701-2706. [CrossRef] [PubMed] 
44. Li, S.; Lo, C.-Y.; Ho, C.-T. Hydroxylated polymethoxyflavones and methylated flavonoids in sweet orange (Citrus sinensis) peel. J. Agric. Food Chem. 2006, 54, 4176-4185. [CrossRef] [PubMed]

45. Stockert, J.C.; Horobin, R.W.; Colombo, L.L.; Blázquez-Castro, A. Tetrazolium salts and formazan products in Cell Biology: Viability assessment, fluorescence imaging, and labeling perspectives. Acta Histochem. 2018, 120, 159-167. [CrossRef] [PubMed]

46. Abate, G.; Ruminiski, P.G.; Kumar, M.; Singh, K.; Hamzabegovic, F.; Hoft, D.F.; Eickhoff, C.S.; Selimovic, A.; Campbell, M.; Chibale, K. New Verapamil Analogs Inhibit Intracellular Mycobacteria without Affecting the Functions of Mycobacterium-Specific T Cells. Antimicrob. Agents Chemother. 2015, 60, 1216-1225. [CrossRef]

47. Stanley, S.A.; Grant, S.S.; Kawate, T.; Iwase, N.; Shimizu, M.; Wivagg, C.; Silvis, M.; Kazyanskaya, E.; Aquadro, J.; Golas, A.; et al. Identification of novel inhibitors of M. tuberculosis growth using whole cell based high-throughput screening. ACS Chem. Biol. 2012, 7, 1377-1384. [CrossRef]

(C) 2020 by the authors. Licensee MDPI, Basel, Switzerland. This article is an open access article distributed under the terms and conditions of the Creative Commons Attribution (CC BY) license (http://creativecommons.org/licenses/by/4.0/). 